\title{
Addressing Health Equity Through Data Collection and Linked Disease Surveillance
}

\author{
Iris Zachary ${ }^{\star 1}$, Jeannette Jackson-Thompson, 1, 3, Emily Leary1 and Eduardo Simoes 1, 2,3 \\ ${ }^{1}$ University of Missouri Health Management and Informatics, Columbia, MO, USA; ${ }^{2}$ Missouri Cancer Registry and Research Center, \\ Columbia, MO, USA; ${ }^{3} \mathrm{MU}$ Informatics Institute, Columbia, MO, USA
}

\section{Objective}

Utilize existing data sets and data sources to address health equity and improve the management of chronic disease

\section{Introduction}

In 2012, half of all adults in the US had one or more chronic health conditions; at least $25 \%$ had two or more chronic health conditions (1). Seven of the top ten causes of death in 2010 were chronic diseases; two of the seven chronic diseases, heart disease and cancer, account almost for over $50 \%$ of all deaths (2). Chronic disease is one of the most costly contributors in healthcare expenditures; once diagnosed many patients must be followed for a lifetime. In lower-income countries chronic disease is now the biggest contributor to mortality (3). Socioeconomic inequalities are a major driver of the chronic disease epidemic (4). Chronic disease in the US, such as cancer, heart disease, renal end stage disease and diabetes are tracked in national datasets but are not linked. Chronic diseases share many risk factors, major risk factors, e.g. tobacco, diet, alcohol, and physical inactivity are already known, their interactions with comorbidities are important and clinical practice indicates that the chronic disease epidemic may be addressed more effectively using a holistic approach. However, this approach has not yet been implemented in disease surveillance activities as data collection is still disease specific. Data collection is still one disease at a time, without connecting our disease surveillance efforts to get better, more complete and encompassing data. Health inequities result in lower quality of healthcare, worse healthcare outcomes for minority racial/ ethnic populations and people with low socioeconomic status, increased direct and indirect healthcare costs, and decreased productivity (5).

\section{Methods}

We identified four chronic diseases that contribute to the majority of death and disease: cancer, diabetes, coronary heart disease, and renal end stage disease. We explored datasets related to each disease to identify comorbidities and other overlapping information and common factors present in all four datasets which allows to look at chronic disease as a whole. We describe the four datasets and the information that we have discovered using this approach and discuss how such information can improve outcomes and potentially reduce inequities. In addition, we demonstrate the advantage to link these data.

\section{Results}

There is an overlap in the data that are collected for the four chronic diseases that contribute to morbidity and mortality in the US and worldwide. We demonstrate that the method of combining datasets will not only enhance data completeness and quality but also increase accuracy for all four datasets and allow for additional research that would not be possible with dataset silos.

\section{Conclusions}

By looking at the four datasets, we were able to identify variations in different racial/ethnic populations, socioeconomic status and risk factors such as tobacco use, obesity and the presence of one or more than one chronic disease. By utilizing data that are already collected and linking datasets, we can capitalize on existing data to support studies that focus on one or more of the diseases and expand the use of previously isolated datasets associated with chronic conditions that contribute to the majority of disease and mortality in the US and worldwide. This approach also opens the door to explore possible connections between chronic diseases that may lead to better understanding of why they go hand-in-hand and to interventions that are culturally appropriate and action-oriented, and can be embedded in the community.

\section{Keywords}

Disease Surveillance; Chronic Disease; Data Linkage

\section{References}

1. Ward BW, Schiller JS, Goodman RA. Multiple chronic conditions among US adults: a 2012 update. Prev Chronic Dis. 2014;11:130389. DOI: http://dx.doi.org/10.5888/pcd11.130389.

2. Centers for Disease Control and Prevention. Death and Mortality. NCHS FastStats Web site. http://www.cdc.gov/nchs/fastats/deaths. htm. Accessed August 25, 2015.

3. Making the link: Chronic diseases and health equity, Fact Sheet Chronic Diseases, 2007 (http://eurohealthnet.eu/sites/eurohealthnet.eu/files/ publications/Fact-Sheet-Chronic\%20diseases\%20and\%20health\%20 equity-V2\%5B2\%5D.pdf). Accessed July 10, 2015.

(4) Health Promotion and disease prevention. Dovec,D., et al. Forum for Public Health Collaboration in South Eastern Europe, 2007

(5) Missouri Health Equity State Snap Shot 2014, (http://www.astho. org/Programs/Health-Equity/Minority-Health-Survey-2014/StateSnapshots/Missouri/). Accessed July 29, 2015.

\section{*Iris Zachary}

E-mail: zacharyi@missouri.edu 